\title{
Modest Blood-Brain Barrier Permeability of the Cyclodextrin Kleptose: Modification by Efflux and Luminal Surface Binding
}

\author{
W.A. Banks, Kory Engelke, Kim M. Hansen, Kristin M. Bullock, and Pericles Calias \\ Geriatric Research and Clinical Center, Veterans Affairs Puget Sound Health Care System, Seattle, Washington (W.A.B., K.M.H., \\ K.M.B.); Division of Gerontology and Geriatric Medicine, Department of Medicine, University of Washington School of Medicine, \\ Seattle, Washington (W.A.B.); The Engelke Group, Keymar, Maryland (K.E.); and Educational Trainers and Consultants, Melrose, \\ Massachusetts (P.C.)
}

Received May 28, 2019; accepted July 16, 2019

\begin{abstract}
Cyclodextrins (CDs) have a variety of uses from acting as excipients to aiding the ability of lipid soluble drugs to cross the blood-brain barrier (BBB). They are being investigated as an active pharmaceutical ingredient, most recently for the treatment of Niemann-Pick disease, a lysosomal storage disease. Cyclodextrins are helpful in animal models and human subjects/ patients afflicted with Neimann-Pick disease, including improving the neurologic component of the disease. The improvement in brain disease by intravenous administration implies that CDs can cross the BBB; however, there are only a few studies that have directly addressed this. In the current studies, multiple-time regression analysis indicated that 2-hydroxypropyl- $\beta$-cyclodextrin [Kleptose (Klep)] radioactively labeled with 14C (C-Klep) crossed the BBB at a slow rate by a nonsaturable mechanism consistent with transcellular diffusion. However, the rate of transport varied greatly by the brain region with no detectable uptake by the spinal cord; additionally, many regions rapidly reached
\end{abstract}

equilibrium between the brain and blood. The presence of a brain-to-blood efflux system was also detected and much of the C-Klep did not completely cross the BBB, but loosely adhered to the luminal surface of brain endothelial cells. Peripheral tissues also took up C-Klep, with the kidney taking up the most, which is consistent with renal clearance. In conclusion, we demonstrated minimal uptake of the $\beta$-cyclodextrin Kleptose by the brain with accumulation being affected by efflux and reversible luminal binding.

\section{SIGNIFICANCE STATEMENT}

This cyclodextrin, which produces therapeutic effects on the central nervous system after peripheral administration, penetrates the BBB poorly. Uptake by the brain to a therapeutic level will likely be difficult to achieve without giving high peripheral doses, bypassing the BBB, or otherwise altering penetration into the brain.

\section{Introduction}

Cyclodextrins (CDs) are cone-shaped oligosaccharides used in an increasing number of therapeutic situations (Loftsson and Brewster, 2010). With a hydrophobic inner surface and a hydrophilic outer surface, CDs are able to bind to hydrophobic molecules or to cap the hydrophobic regions of molecules, resulting in a more water-soluble complex.

Originally, CDs were used as excipients, improving the delivery of small molecules such as prostaglandins (Davis and Brewster, 2004). This excipient property has been extended to peptides and proteins, with the CD capable of binding to the functional groups of the amino acids (Varca et al., 2010). CDs have been postulated to aid in the systemic delivery of peptides and proteins after oral or nasal administration (Soares et al., 2007). Nasal delivery when administered high at the level of the cribriform plate can result in

This work was funded by the the US Department of Veterans Affairs. https://doi.org/10.1124/jpet.119.260497. uptake by the brain (Frey, 2002). Peptides combined with CDs can have an altered distribution pattern in the brain, raising the possibility that they may be useful in targeting specific regions (Nonaka et al., 2008, 2012).

More recently, CDs have been identified as having direct pharmacologic actions. One such effect is that CDs facilitate the clearing of cholesterol from cells. In both animal models and patients with Niemann-Pick disease (an autosomal recessive lysosomal storage disease that results in accumulation of cholesterol and glycosphingolipids in cells), 2-hydroxypropyl$\beta$-cyclodextrin [trademarked as Kleptose (Klep)], results in significant removal of cholesterol from the liver and decreased hepatosplenomegaly (Davidson et al., 2009; Liu et al., 2009; Matsuo et al., 2013). In mice, peripheral administration of the CD results in reduced intraneuronal storage of cholesterol and glycoshingolipids, decreased neuroinflammation, less neurodegeneration, and significant prolongation of life (Davidson et al., 2009; Liu et al., 2009). In humans, peripheral administration resulted in measurable but less spectacular improvements in brain function (Matsuo et al., 2013).

ABBREVIATIONS: BBB, blood-brain barrier; CD, cyclodextrin; C-Klep, 2-hydroxypropyl- $\beta$-cyclodextrin radioactively labeled with 14C [(2-hydroxy[2-14C]propyl)- $\beta$-cyclodextrin]; CNS, central nervous system; I-Alb, albumin radioactively labeled with $125 \mathrm{l} ; \%$ Inj/g, percentage of the injected dose taken up per gram of tissue; $K_{\mathrm{i}}$, unidirectional influx rate; Klep, Kleptose (2-hydroxypropyl- $\beta$-cyclodextrin); Tc-Alb, albumin radioactively labeled with 99mTc. 

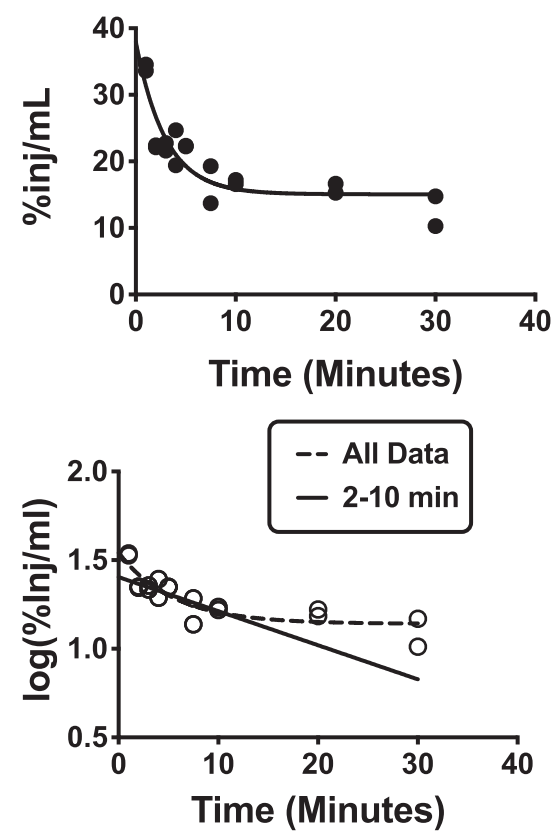

Fig. 1. Clearance of C-Klep from circulation after intravenous injection: (top panel) clearance of C-Klep expressed as the percentage of the injected dose taken up per milliliter (\%Inj/ml) vs. time showed two phases; (bottom panel) clearance using $\log (\% \mathrm{Inj} / \mathrm{ml})$ vs. time shows early distribution phase with half-time disappearance of 15.7 minutes followed by a plateau. $N=18$ for both panels.

The aforementioned studies have shown that peripheral administration of CDs can result in improvement of central nervous system (CNS) symptoms. This, in turn, would suggest that CDs have the potential to cross the blood-brain barrier (BBB) (Calias, 2017). Several studies have shown that drugs complexed with CD can enter the CNS as well as cross other membrane barrier membranes, including the BBB (Gil et al., 2012; Conceicao et al., 2018). However, there are few studies available that have examined the ability of CDs themselves to cross the BBB. Using an in vitro model of the BBB consisting of primary brain endothelial cells, Monnaert et al. (2004) found that several CDs crossed the BBB. The rate of passage varied over 4-fold and the effects of modifications with methylation or hydroxypropylation were variable and not predictable. In vivo studies in mice have found large distribution volumes in the brain after peripheral administration of radioactive $\mathrm{CD}$ (Pontikis et al., 2013). Because those distribution volumes did not increase over time, as is typical of blood-to brain transport, it was proposed that the uptake by the brain represented binding to the luminal surface of brain endothelial cells, a rare but documented cause of false positives for the gold standard techniques that they used (Triguero et al., 1990; Maness et al., 1994).

Here, we quantified the degree to which intravenously administered radioactive Klep [2-hydroxy-[2-14C]propyl)$\beta$-cyclodextrin] (C-Klep) can cross from blood into whole brain, brain regions, and spinal cord. We also evaluated its ability to cross in the brain-to-blood direction, its uptake by peripheral tissues, its reversible binding to the luminal surface of the $\mathrm{BBB}$, and its sequestration by brain endothelial tissues.

\section{Materials and Methods}

Radioactive Materials. Kleptose [(2-hydroxy-[2-14C]propyl)$\beta$-cyclodextrin] that was radioactively labeled with $14 \mathrm{C}$ (C-Klep) was obtained from Institute of Isotopes Co Ltd. (Budapest, Hungry).

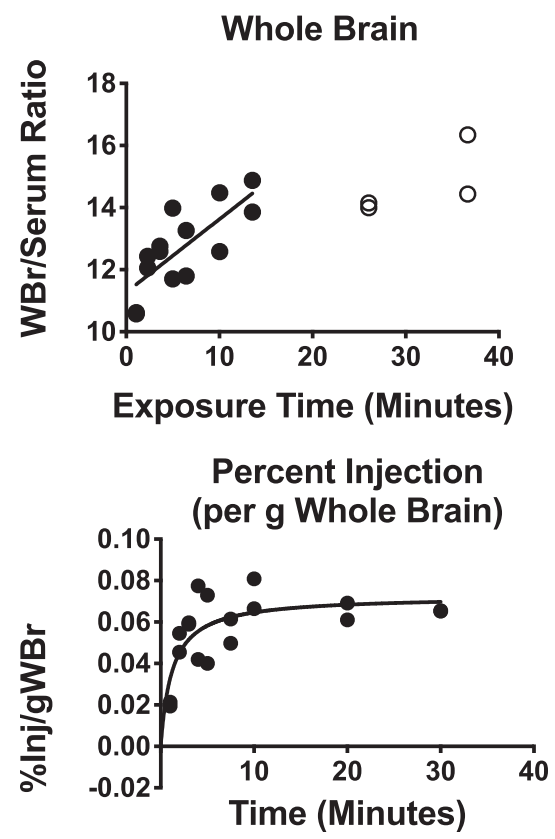

Fig. 2. Uptake of C-Klep by whole brain: (top panel) uptake was linear for the first 15-20 minutes after intravenous injection, yielding a unidirectional influx rate of $0.236 \pm 0.057 \mu \mathrm{l} / \mathrm{g} \cdot \mathrm{min}$; (lower panel) uptake expressed as \%Inj/g followed a hyperbolic pattern, reaching a theoretical maximum of $.072 \pm 0.006 \%$ Inj/g. $N=18$ for both panels.

Specific activity was $(0.251 \mathrm{MBq} / \mathrm{mg})$. Bovine serum albumin was obtained from Sigma-Aldrich (St. Louis, MO) and labeled with 125I using the chloramine-T method [albumin radioactively labeled with $125 \mathrm{I}$ (I-Alb) or albumin radioactively labeled with $99 \mathrm{mTc}$ (Tc-Alb)] and purified on a column of Sephadex G-10.

Tissue Uptake. Multiple-time regression analysis (Blasberg et al., 1983; Patlak et al., 1983): male mice averaging about $30 \mathrm{~g}$ in body weight were anesthetized with an intraperitoneal injection of urethane, with the left carotid artery and right jugular vein exposed. Mice were given an injection into the jugular vein of $200 \mu \mathrm{l}$ of lactated Ringer's solution containing $106 \mathrm{dpm}$ of a 14C-labeled Kleptose. Blood from the carotid artery was obtained and the mice were then immediately decapitated at

TABLE 1

Unidirectional influx rates for C-Klep

\begin{tabular}{lccrll}
\hline Tissue & $K_{\text {i }} \pm$ S.E. & $V_{\text {i }} \pm$ S.E. & $n$ & $R^{2}$ & $P<$ \\
\hline & $\mu l / g \cdot \min$ & $\mu l / g$ & & & \\
Olfactory bulb & $0.413 \pm 0.137$ & $29.5 \pm 2.2$ & 18 & 0.361 & 0.01 \\
Whole brain & $0.235 \pm 0.057$ & $11.3 \pm 0.42$ & 14 & 0.586 & 0.005 \\
Frontal cortex & $0.062 \pm 0.018$ & $9.05 \pm 0.30$ & 18 & 0.410 & 0.005 \\
Parietal cortex & $0.110 \pm 0.040$ & $10.6 \pm 0.66$ & 18 & 0.317 & 0.05 \\
Occipital cortex & $0.708 \pm 0.175$ & $11.7 \pm 1.3$ & 14 & 0.578 & 0.005 \\
Hippocampus & $0.101 \pm 0.035$ & $10.4 \pm 0.6$ & 17 & 0.356 & 0.05 \\
Hypothalamus & $0.757 \pm 0.155$ & $16.4 \pm 1.8$ & 16 & 0.629 & 0.001 \\
Thalamus & $0.235 \pm 0.059$ & $8.57 \pm 0.43$ & 14 & 0.567 & 0.005 \\
Striatum & $0.202 \pm 0.058$ & $12.2 \pm 0.9$ & 18 & 0.434 & 0.005 \\
Cerebellum & $0.330 \pm 0.095$ & $13.4 \pm 0.7$ & 14 & 0.499 & 0.005 \\
Midbrain & $0.382 \pm 0.096$ & $10.6 \pm 0.7$ & 14 & 0.569 & 0.005 \\
Pons medulla & $0.156 \pm 0.033$ & $17.1 \pm 0.5$ & 18 & 0.584 & 0.001 \\
Cervical SC & $0.365 \pm 0.113$ & $15.9 \pm 1.8$ & 18 & 0.403 & 0.005 \\
Thoracic SC & $0.0893 \pm 0.019$ & $5.98 \pm 0.31$ & 18 & 0.584 & 0.001 \\
Lumbar SC & $0.177 \pm 0.052$ & $9.95 \pm 0.85$ & 18 & 0.421 & 0.005 \\
Spleen & $0.654 \pm 0.181$ & $111 \pm 3$ & 18 & 0.449 & 0.005 \\
Lung & $26.9 \pm 7.0$ & $236 \pm 18$ & 6 & 0.785 & 0.05 \\
Kidney & $7.72 \pm 2.63$ & $200 \pm 38$ & 16 & 0.381 & 0.05 \\
Liver & $7.97 \pm 2.75$ & $66.2 \pm 7.0$ & 6 & 0.677 & 0.05 \\
Heart & $0.537 \pm 0.347$ & $193 \pm 6$ & 18 & 0.130 & $\mathrm{NS}$ \\
G Muscle & $6.14 \pm 1.14$ & $55.8 \pm 6.3$ & 12 & 0.745 & 0.001 \\
\hline
\end{tabular}

G, gastrocnemius; NS, not statistically significant; SC, spinal cord. 
the time points of $1,2,3,4,5,7.5,10,20$, and 30 minutes with $n=2 /$ time point for a total of 18 . The brain (frontal cortex, parietal cortex, occipital cortex, hippocampus, hypothalamus, thalamus, striatum cerebellum, midbrain, pons medulla, and olfactory bulb) and spinal cord (cervical, thoracic, and lumbar) were dissected and weighed, serum was obtained as previously described, and levels of radioactivity were determined. The results were expressed as brain region/serum ratio in units of microliters per gram. The values for whole brain were calculated by summing the values for the aforementioned brain regions (the olfactory bulb). The peripheral tissues, or portions thereof (kidney, spleen, heart, gastrocnemius muscle, liver, and lung), were obtained and weighed, with the levels of radioactivity expressed as microliters per gram. The tissue/serum ratios were plotted against the exposure time (in minutes), which was calculated as

$$
\operatorname{Expt}=\frac{\left[\int_{0}^{\mathrm{t}} \mathrm{Cp}(\tau) \mathrm{d} \tau\right]}{\mathrm{Cpt}}
$$

where Expt is the exposure time; $t$ is the time between the intravenous injection and sampling; $\mathrm{Cp}$ is the counts per minute per milliliter of arterial serum; Cpt is the counts per minute per milliliter of arterial serum at time $t$; and $\tau$ is the dummy variable for time. The slope of the linear portion of the relation of tissue/serum ratio versus exposure time measured the unidirectional influx rate $\left(K_{\mathrm{i}}\right)$ in units of microliters per grams per minute.

Other mice $(n=20)$ were given injections as previously described, except that one-half of the mice $(n=10)$ had $30 \mathrm{mg}$ of unlabeled Kleptose included in the jugular injection. Tissues were collected as previously described, with the results expressed as microliters per gram, and groups were compared by Student's $t$ test. Other mice anesthetized with urethane were given an injection into the jugular vein of lactated Ringer's solution containing $106 \mathrm{cpm}$ of I-Alb. Brain and peripheral tissues were obtained and weighed, and radioactive levels were determined as previously described at 1,2, 5, 10, 20, and 30 minutes after the injection. Since these values did not change over time, they were combined across time and the mean tissue/serum ratio subtracted from the ratio for C-Klep and multiplied by 100 and by the dose of C-Klep injected to yield the percentage of the injected dose taken up per gram of tissue $(\%$ Inj/g). Other mice $(n=19)$ anesthetized with urethane were given an injection into the jugular vein of $200 \mu \mathrm{l}$ of lactated Ringer's solution containing $106 \mathrm{dpm}$ of C-Klep and $106 \mathrm{cpm}$ of Tc-Alb. Ten minutes after the intravenous injection, the abdomen and thorax were opened, blood was collected from a nick in the abdominal
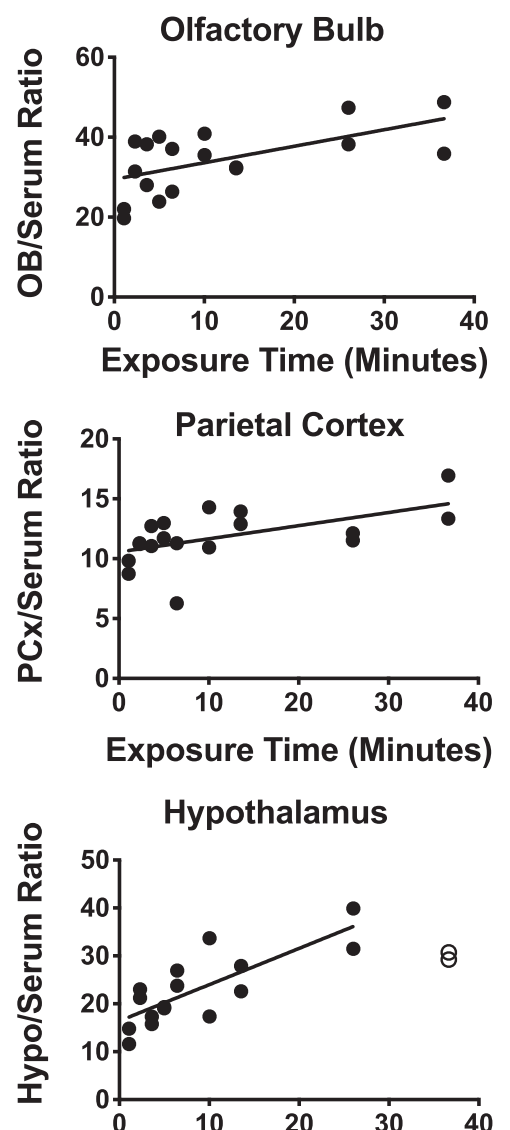

Exposure Time (Minutes)

Striatum

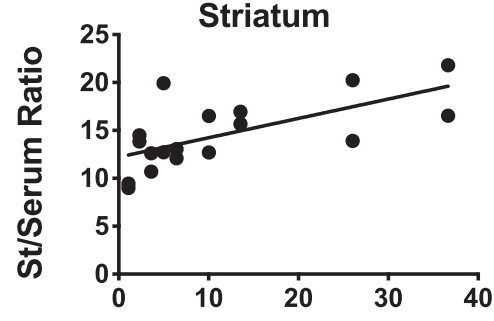

Exposure Time (Minutes)
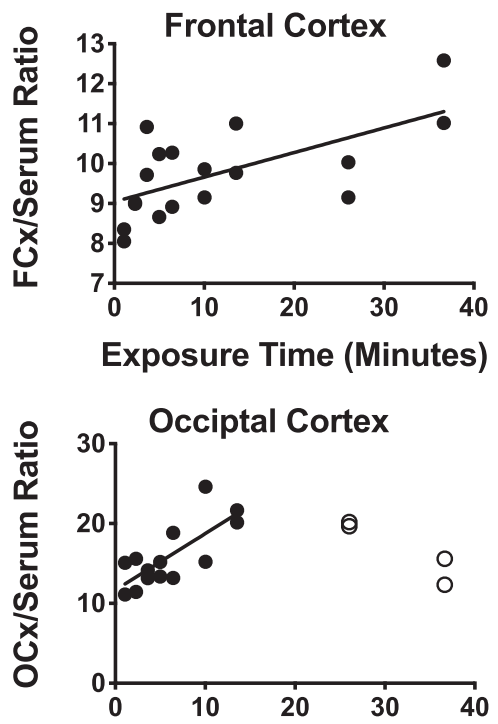

Exposure Time (Minutes)

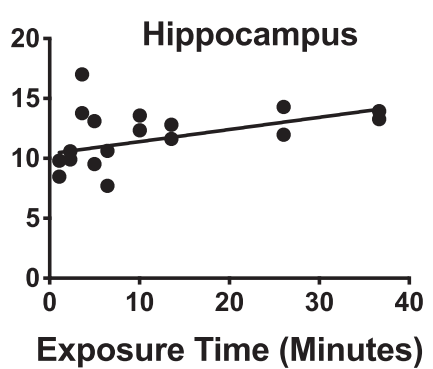

Fig. 3. Uptake of C-Klep by brain region. All regions took up C-Klep, but uptake varied by over 10 -fold among the regions. The hypothalamus with a $K_{\mathrm{i}}$ value of $0.757 \pm 0.155 \mu \mathrm{l} / \mathrm{g} \cdot \mathrm{min}$ had the fastest uptake and the region with the slowest uptake was the frontal cortex with a $K_{\mathrm{i}}$ value of $0.062 \pm 0.018 \mu \mathrm{l} / \mathrm{g} \cdot \mathrm{min}$. $N=18$ per panel.

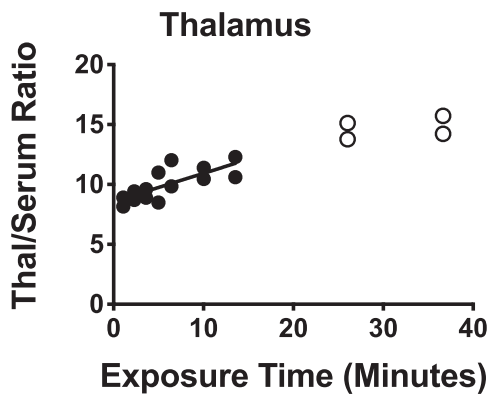


aorta, and the jugular veins were severed. In 10 mice, $20 \mathrm{ml}$ of cold lactated Ringer's solution was infused into the left ventricle of the heart, thus washing out the vascular space of the brain, whereas nine mice did not receive washout. Levels of $99 \mathrm{mTc}$ and $14 \mathrm{C}$ were determined in whole brain and arterial serum and expressed as brain/serum ratios.

Brain-to-Blood Passage. Brain-to-blood efflux rates were assessed by the intracerebroventricular injection method (Banks and Kastin, 1986; Banks et al., 1986). Mice were anesthetized with an intraperitoneal injection of $0.15 \mathrm{ml}$ of $40 \%$ urethane (Sigma-Aldrich). The scalp was removed and a hole was made into the skull $(0.5 \mathrm{~mm}$ posterior and $1 \mathrm{~mm}$ lateral to the bregma) and $1 \mu \mathrm{l}$ of lactated Ringers solution containing $2.5 \times 10^{4} \mathrm{dpm}$ of C-Klep with or without unlabeled Klep $(0.15 \mathrm{mg} /$ mouse $)$ was injected into the lateral ventricle of the brain using a $1.0 \mu \mathrm{l}$ Hamilton syringe. Ten minutes after the intracerebroventricular injection, blood was obtained from the carotid artery and the mice were decapitated. The whole brain was removed and weighed. The amount of C-Klep available for transport at $\mathrm{t}=0$ was estimated in mice that had been overdosed with urethane and had been dead for 10-20 minutes before receiving C-Klep (Banks and Kastin, 1989; Banks et al., 1997). Solvable (1.5 ml) was added to the brain or to $0.50 \mathrm{ml}$ of the arterial serum and incubated overnight at $60^{\circ} \mathrm{C}, 15 \mathrm{ml}$ of ecoscint was added, and each sample was counted for 60 minutes using a beta counter. The amount of C-Klep that was transported out of the brain $(\% \mathrm{~T})$ was determined with the equation:

$$
\% \mathbf{T}=\left[100(\mathrm{cpm} / \mathrm{g})_{0}-(\mathrm{cpm} / \mathrm{g})_{10}\right] /(\mathrm{cpm} / \mathrm{g})_{0}
$$

where 0 is the mean level of cpm/g for the $t=0$ group and $(\mathrm{cpm} / \mathrm{g}) 10$ is the individual mouse's level of $\mathrm{cpm} / \mathrm{g}$ at $\mathrm{t}=10$ minutes. The appearance in blood of C-Klep 10 minutes after intracerebroventricular injection was expressed as the counts per minute in a milliliter of arterial serum divided by the amount injected multiplied by 100 and designated as the percentage of the injected dose taken up per milliliter.

Octanol/Buffer Coefficient. About 40,000 dpm of C-Klep were placed into a test tube containing $500 \mu \mathrm{l}$ of octanol and $500 \mu \mathrm{l}$ of PBS was placed on top. The two phases were vigorously mixed for 1 minute and the test tube was centrifuged for 10 minutes at $4^{\circ} \mathrm{C}$. An aliquot from each of the two phases was carefully removed and the level of radioactivity was determined. The results were expressed as the ratio of the dpm in the octanol phase divided by the dpm in the buffer phase.

Capillary Depletion. The capillary depletion method was used to separate cerebral capillaries and vascular components from brain parenchyma. Mice were anesthetized with an intraperitoneal injection of $0.15 \mathrm{ml}$ of $40 \%$ urethane. Mice received an injection of $1 \times 10^{6}$ cpm Tc-Alb with $2 \times 10^{6} \mathrm{dpm}$ C-Klep in $0.2 \mathrm{ml}$ of lactated Ringer's solution, and 10 minutes later five mice underwent washout as outlined previously and four did not have washout. The whole brain was homogenized in glass with physiologic buffer and mixed thoroughly with $26 \%$ dextran. The homogenate was centrifuged at $5400 \mathrm{~g}$ for 15 minutes at $4^{\circ} \mathrm{C}$. The pellet (containing the capillaries) and the supernatant (representing the brain parenchymal/interstitial fluid space) were carefully separated. Radioactivity levels in the capillary pellet, brain supernatant, and arterial serum were determined for both Tc-Alb and C-Klep and expressed as capillary/serum and brain parenchyma/serum ratios. The C-Klep ratios were corrected for vascular contamination by subtracting the corresponding ratios for Tc-Alb; these results are reported as the delta capillary/serum ratio and the delta brain parenchyma/serum ratio.

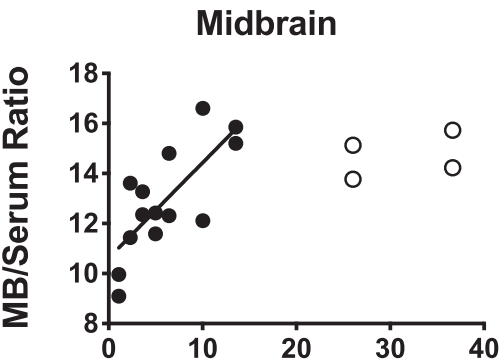

Exposure Time (Minutes)

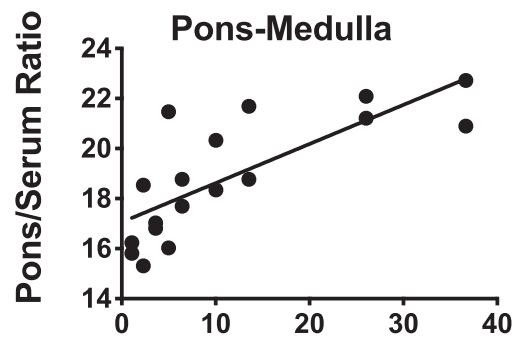

Exposure Time (Minutes)

Thoracic Spinal Cord

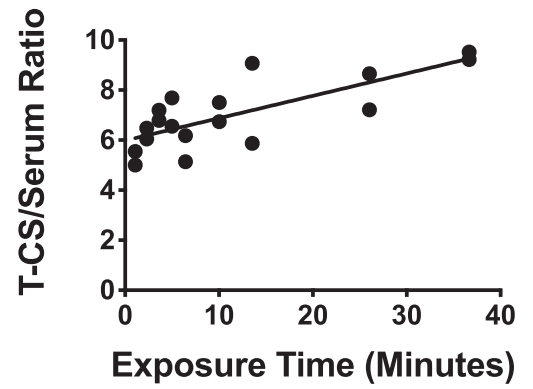

Cerebellum
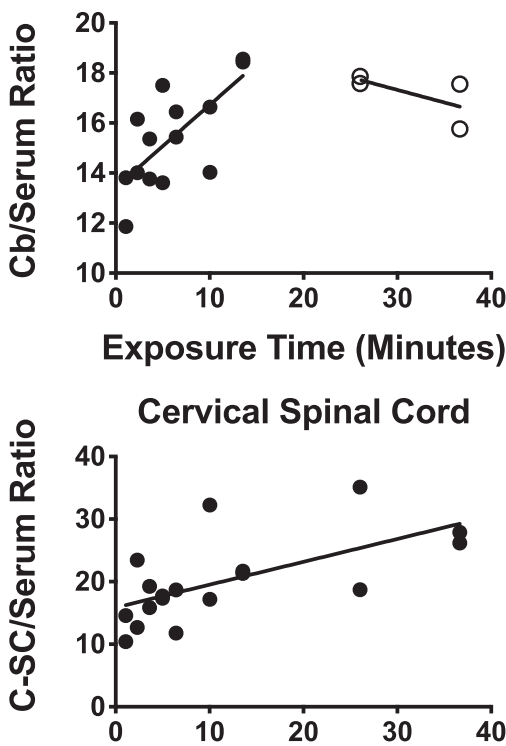

Fig. 3. Continued.

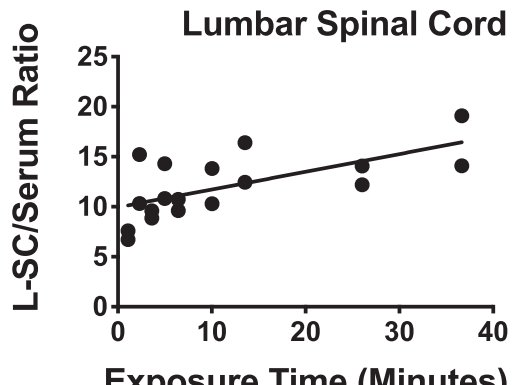

Exposure Time (Minutes) 
Statistics. Calculations and comparisons of regression lines were performed using Prism 6.0 (GraphPad Inc., San Diego, CA). Two mean values were compared by Student's $t$ test and more than two mean values were compared by ANOVA followed by Newman-Keuls range test. Mean values are reported with their S.E. term and $n$.

\section{Results}

Figure 1 shows the relation between serum concentration and time after intravenous injection. An early phase (2-10 minutes) with a half-life disappearance of 15.7 minutes was followed by an equilibrium phase. The volume of distribution for the first 10 minutes after injection, as calculated from the antilog of Fig. 1 (lower panel, linear line), was $24.8 \%$ of the injected dose taken up per milliliter (or about $4.0 \mathrm{ml}$ ).

C-Klep crossed the BBB with a $K_{\mathrm{i}}$ value of $0.236 \pm 0.057 \mu \mathrm{l} / \mathrm{g} \cdot \mathrm{min}$ (Fig. 2, upper panel; Table 1). The percentage of the intravenously administered dose taken up per gram of brain formed a hyperbolic curve with time and had a theoretical maximal value of $0.072 \pm 0.006$, reaching steady state about 10 minutes after intravenous injection (Fig. 2, lower panel).

C-Klep entered all regions of the central nervous system, including the olfactory bulb and all regions of the spinal cord
(Fig. 3). The $K_{\mathrm{i}}$ value was calculated using only the linear portion of the tissue/serum versus exposure time ratio; the filled circles in Fig. 3 show the data points used to calculate the $K_{\mathrm{i}}$ value, while the open circles were excluded because they departed from linearity. Brain regions varied over 10 -fold in their rates of uptake for C-Klep. The region with the fastest uptake was the hypothalamus with a $K_{\mathrm{i}}$ value of 0.757 $\pm 0.155 \mu \mathrm{l} / \mathrm{g} \cdot \mathrm{min}$ and the region with the slowest uptake was the frontal cortex with a $K_{\mathrm{i}}$ value of $0.062 \pm 0.018 \mu \mathrm{l} / \mathrm{g} \cdot \mathrm{min}$. The occipital cortex, hypothalamus, cerebellum, thalamus, and midbrain reached equilibrium during the course of the study, whereas the other brain regions continued to accumulate C-Klep throughout the study.

Tissue/serum ratios correlated with exposure for all peripheral tissues except heart, indicating active accumulation by these tissues (Fig. 4). Lung had the fastest uptake and quickly reached steady state. Spleen had the slowest uptake. Of the tissues with measurable accumulation, only spleen and kidney did not reach steady state during the study.

To express results as \% Inj/g, we needed to measure and correct regions for vascular space. The results for the vascular spaces are shown in Table 2 . Figure 5 shows the results for $\% I n j / g$. Olfactory bulb and hypothalamus had the highest uptake rates,

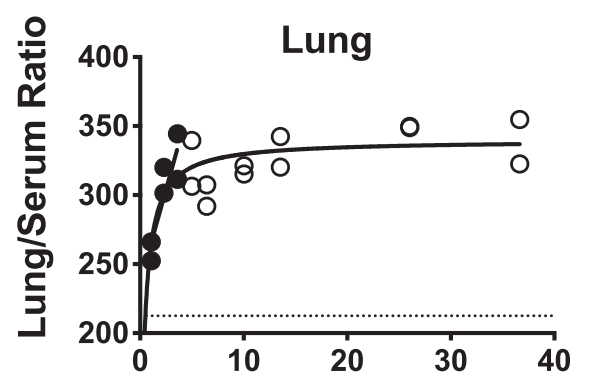

Exposure Time (Minutes)
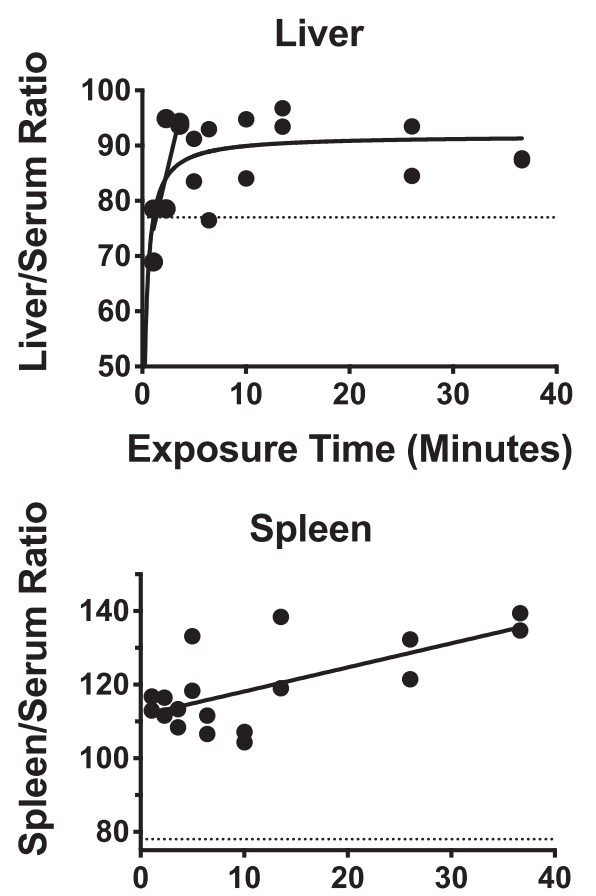

Exposure Time (Minutes)

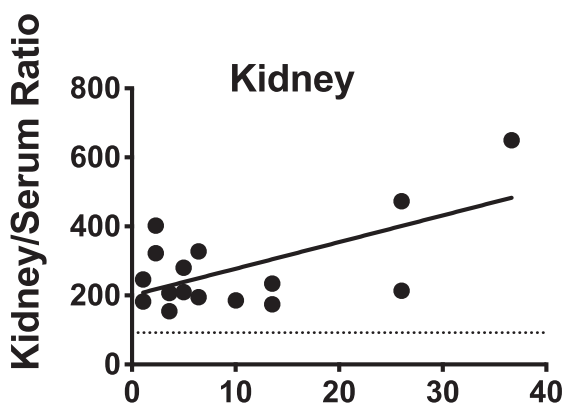

Exposure Time (Minutes)
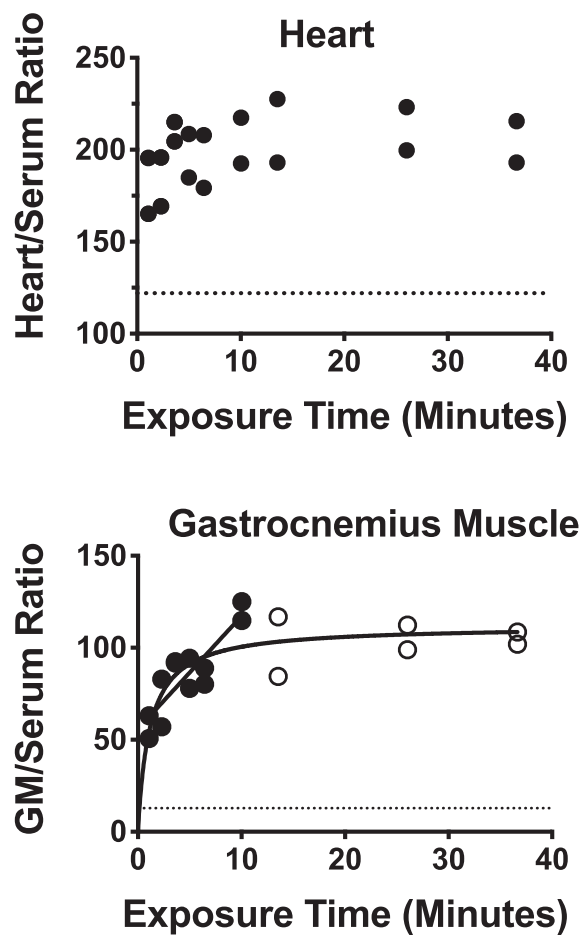

Fig. 4. Uptake of C-Klep by peripheral tissues. All tissues showed a significant correlation between tissue/serum ratios vs. time except for heart. The dashed line shows the vascular space for the tissue as measured by radioactive albumin. $N=18 \mathrm{per}$ panel. 
TABLE 2

Vascular space as measured by albumin

\begin{tabular}{lr}
\hline Tissue & \multicolumn{1}{c}{ Value $^{a}$} \\
\hline & $\mu l / g$ \\
Olfactory bulb & $19.6 \pm 1.7$ \\
Whole brain & $11.3 \pm 0.8$ \\
Frontal cortex & $9.5 \pm 0.7$ \\
Parietal cortex & $10.0 \pm 1.3$ \\
Occipital cortex & $13.7 \pm 1.5$ \\
Hippocampus & $10.2 \pm 0.5$ \\
Hypothalamus & $14.2 \pm 1.5$ \\
Thalamus & $8.1 \pm 0.3$ \\
Striatum & $12.1 \pm 2.0$ \\
Cerebellum & $13.7 \pm 1.9$ \\
Midbrain & $12.4 \pm 0.9$ \\
Pons medulla & $14.2 \pm 0.7$ \\
Cervical SC & $23.9 \pm 3.3$ \\
Thoracic SC & $8.6 \pm 1.0$ \\
Lumbar SC & $11.5 \pm 2.2$ \\
Spleen & $78.0 \pm 5.2$ \\
Lung & $212.5 \pm 26.8$ \\
Kidney & $92.0 \pm 4.9$ \\
Liver & $77.1 \pm 4.5$ \\
Heart & $122.3 \pm 3.3$ \\
G Muscle & $12.8 \pm 0.8$ \\
\hline
\end{tabular}

G, gastrocnemius; SC, spinal cord.

${ }^{a}$ Mean \pm S.E., $n=6$.

whereas frontal cortex had the least. For peripheral tissues, kidney had the highest uptake, whereas liver had the lowest value for \%Inj/g. Although heart did not have a measurable $K_{\mathrm{i}}$ value, its tissue/serum values for C-Klep exceeded those for vascular space, and thus had a measurable value for $\%$ Inj/g; likely it reached equilibrium so quickly that the $K_{\mathrm{i}}$ value could not be measured with the approach taken here. The \%Inj/g corrected for albumin space was negative for the three spinal cord regions $(n=18)$ : cervical: $-0.1 \pm 0.04$; thoracic $-0.04 \pm 0.01$; and lumbar: $-0.004 \pm 0.02$.

Neither whole brain nor any brain region exhibited evidence for inhibition with the dose of $30 \mathrm{mg} /$ mouse of unlabeled Klep (Fig. 6). Serum showed a trend toward a decrease and kidney showed a dramatic uptake.

The octanol/buffer coefficient is a measure of lipid solubility and correlates with the ability of substances to cross the BBB by the mechanism of transcellular diffusion (el Tayar et al., 1991). The octanol/buffer coefficient for C-Klep was $(1.3 \pm 0.1) 10^{-3}$ $(n=6)$, in which the log value is -2.89 . The results for washout of whole brain are shown in Table 3. The Tc-Alb results showed that the washout removed $>90 \%$ of the vascular contents. However, the brain/serum ratio for C-Klep decreased with washout from 36.1 to $4.4 \mu \mathrm{l} / \mathrm{g}$, much more than was expected from washout of vascular contents. Two common explanations for such large decreases are a rapid brain-to-blood efflux mechanism and reversible binding to the luminal surface of brain endothelial cells. To determine whether the remaining C-Klep was sequestered by the brain endothelial cells or had completely crossed the BBB, we performed capillary depletion. The delta capillary/ serum ratio after washout was $0.027 \pm 0.007 \mu \mathrm{l} / \mathrm{g}(n=5)$ and the delta brain parenchyma/serum ratio was $1.07 \pm 0.16(n=5)$, demonstrating that some C-Klep completely crossed the BBB to enter the brain.

Ten minutes after intracerebroventricular injection, $36.6 \pm 3.6 \% \mathrm{~T}(n=9)$ of the C-Klep had been transported from brain (Fig. 7, left panel). The value for the group receiving unlabeled Klep was $50 \pm 2.0 \% \mathrm{~T}(n=10)$. This was an increase

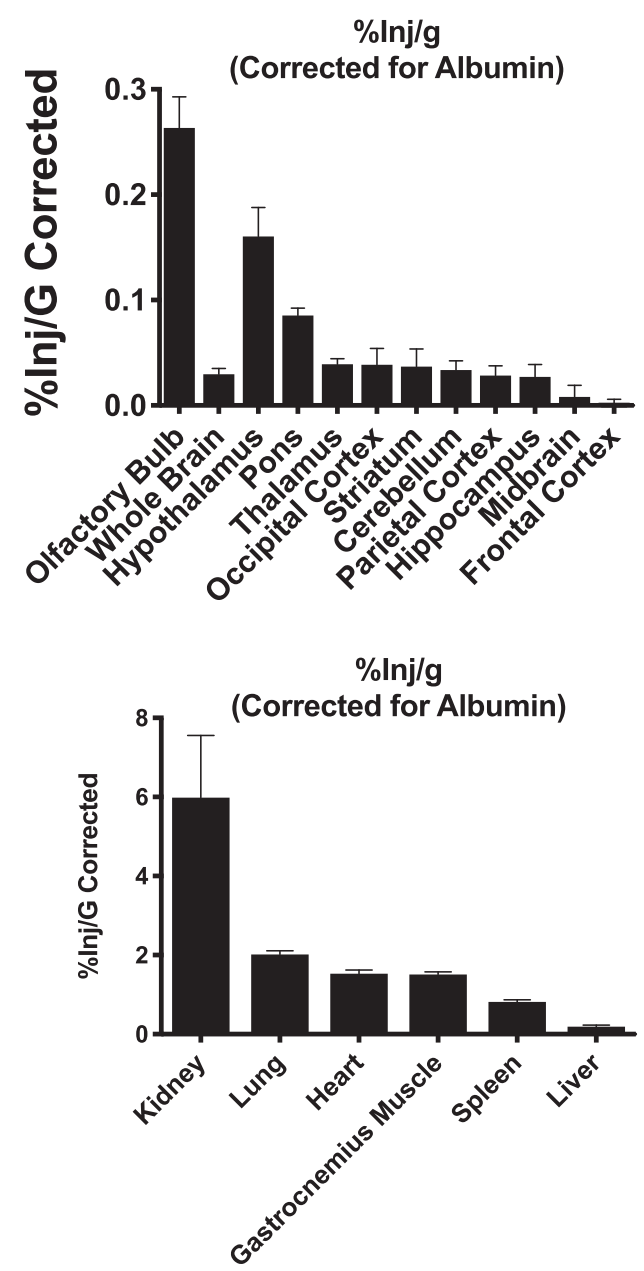

Fig. 5. The percentage of the injected dose taken up per gram of tissue: (upper panel) results for brain regions; (lower panel) results for peripheral tissues. $N=18$ per group.

of $37 \%$ and was statistically significant from the group receiving C-Klep only: $\mathrm{t}=3.36, \mathrm{df}=17, P<0.005$. C-Klep appeared in arterial serum after intracerebroventricular injection, confirming its brain-to-blood movement (Fig. 7, right). For the C-Klep-only group, $2.21 \pm 0.19(n=9)$ percent of the intracerebroventricularly injected dose appeared in blood and for the C-Klep + unlabeled Klep group, the value was $2.78 \% \pm 0.21 \%(n=10)$ of the intracerebroventricularly injected dose. This was an increase of $26 \%$ and was not statistically significant: $0.1<P<0.05$.

\section{Discussion}

These studies were designed to investigate the uptake of a cyclodextrin by and across the BBB. We evaluated C-Klep for uptake by various regions of the brain and by peripheral tissues, for loose adherence to the luminal surface of brain capillaries and brain-to-blood efflux. We found that uptake was nonsaturable, suggesting that uptake was by the mechanism of transcellular diffusion. The octanol/buffer coefficient measured for C-Klep shows it to be a highly water soluble compound, and the $K_{\mathrm{i}}$ value for whole brain is similar to that of water soluble peptides of similar size that cross the BBB by the mechanism of transcellular diffusion (Banks et al., 1984; Banks and Kastin, 1985). The unlabeled dose of Klep did not have a statistically significant effect on C-Klep uptake for any 


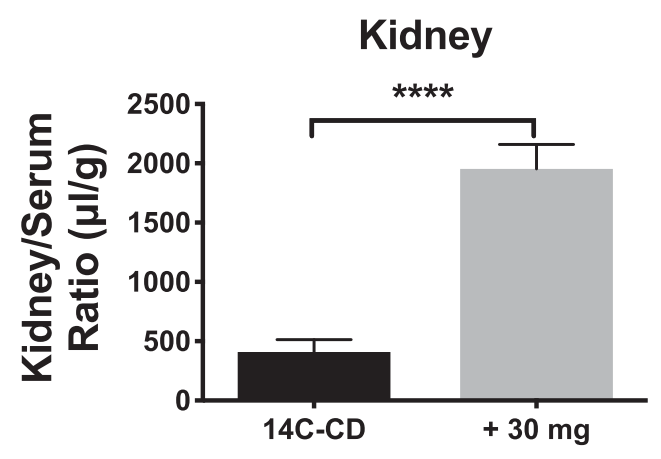

Serum

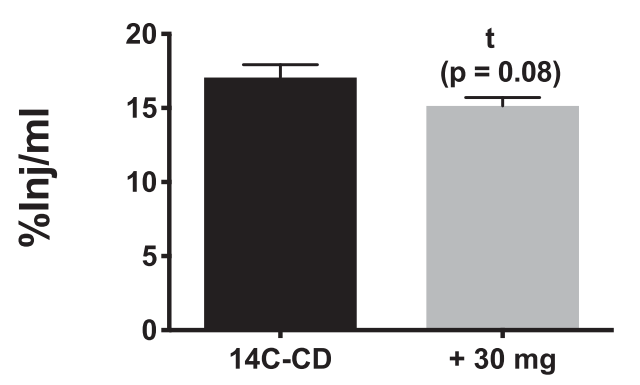

Liver

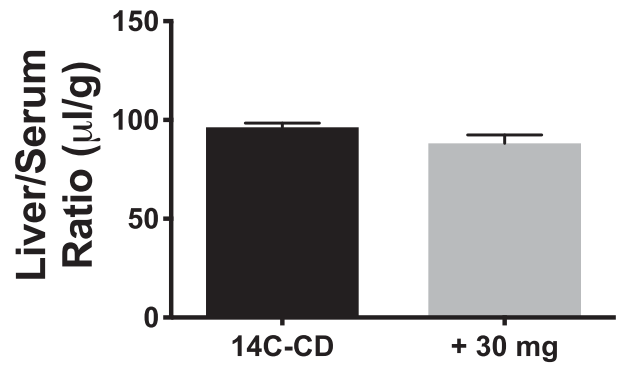

Whole Brain

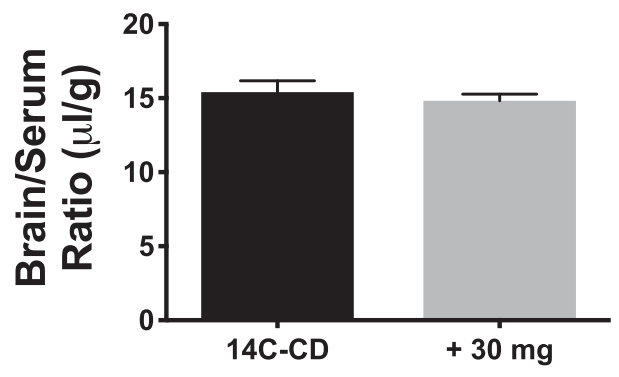

Fig. 6. Effects of unlabeled Kleptose on uptake by brain, serum, liver, and kidney: no evidence of saturable uptake was found for any tissue; however, a paradoxic increase in kidney and a trend for a decrease in serum was found. $N=8$ for kidney; $N=10$ for all other groups. region of the central nervous system. However, the uptake rate varied by 10 -fold among brain regions, whereas the spinal cord regions after correction for vascular space showed no uptake of C-Klep. This large range among brain regions for values of $K_{\mathrm{i}}$ and the lack of uptake by the spinal cord is not typical of transcellular diffusion. Another unusual feature of the brain uptake curve was that whole brain and several brain regions rapidly came to steady-state levels. We conclude that whereas transcellular diffusion is an important mechanism by which C-Klep crosses the BBB, other modifying mechanisms are also likewise at work.

One such modifying factor would be the presence of a brainto-blood transport system. Such systems are classic causes of blood-to-brain curves rapidly reaching equilibrium, and in extreme cases even giving the appearance that a substance cannot cross the BBB (Banks and Kastin, 1984; Kastin et al., 2002). If the activity of such a system varied regionally as has been shown for the BBB efflux transporter P-glycoprotein (Kannan et al., 2017), it could explain differences in retention among brain regions. We tested for an efflux system by injecting C-Klep into the lateral ventricle of the brain and determining the rate of efflux out of the brain. We found that by 10 minutes, $36.6 \%$ of the intracerebroventricularly injected dose available for transport had left the brain; this gives a half-time clearance from the brain of 15.2 minutes. This is much faster than the expected half-life of about 45 minutes if clearance had been only by the mechanism of cerebrospinal fluid reabsorption (Banks et al., 1988), thus implying a brainto-blood transporter. That C-Klep was exiting the CNS and entering the bloodstream is confirmed by the appearance of radioactivity in blood after the intracerebroventricular injection. The inclusion of unlabeled Klep in the intracerebroventricular injection paradoxically increased the rate of efflux, rather than inhibiting it as expected in the presence of a brainto-blood transporter. This paradox could be caused if sequestration by brain tissue is saturable or otherwise limited.
Another factor that would modify uptake would be if either the C-Klep was not fully transported across the BBB but sequestered by the capillary or if the C-Klep is only loosely adhering to the luminal surface of the capillary. The latter is a rare but documented cause of overestimation of the rate of transport across the BBB (Triguero et al., 1990; Maness et al., 1993). Furthermore, a previous study has suggested that cyclodextrins bind to the luminal surface of capillaries with limited passage across the BBB (Pontikis et al., 2013). We used the capillary depletion method with and without washout to divide the extracellular space into three compartments: C-Klep reversibly adhering to the luminal surface, C-Klep sequestered by brain capillaries, and C-Klep that had completely crossed the BBB. We found the majority of C-Klep was loosely adhering to the luminal surface of the capillaries, with no evidence of sequestration by the capillaries, and a small amount of C-Klep that had completely crossed the BBB.

The finding that uptake is by a nonsaturable mechanism is important in the therapeutic use of Klep. The percentage of the peripherally administered dose that enters the brain should be constant regardless of dose given. This simplifies the calculation of a dose that would achieve a therapeutic effect but be below that of toxicity. It also simplifies the calculation of the amount of drug in the CNS after any peripheral dose. For example, here, as a test for inhibition, we gave a dose of $1 \mathrm{~g} / \mathrm{kg}$ (or about $30 \mathrm{mg} / \mathrm{mouse}$ ). Since whole brain accumulated about $0.06 \%$ of the administered dose, this would give a concentration of $21.6 \mu \mathrm{g}$ of drug per gram of whole brain.

\section{TABLE 3}

Brain/serum ratios for C-Klep with and without washout of the brain vascular space

Mean \pm S.E.; units in microliters per gram.

\begin{tabular}{lcc}
\hline & No Washout $(n=9)$ & Washout $(n=10)$ \\
\hline Tc-Alb & $9.7 \pm 0.2$ & $0.8 \pm 0.1$ \\
C-Klep & $36.1 \pm 2.0$ & $4.4 \pm 0.2$ \\
\hline
\end{tabular}




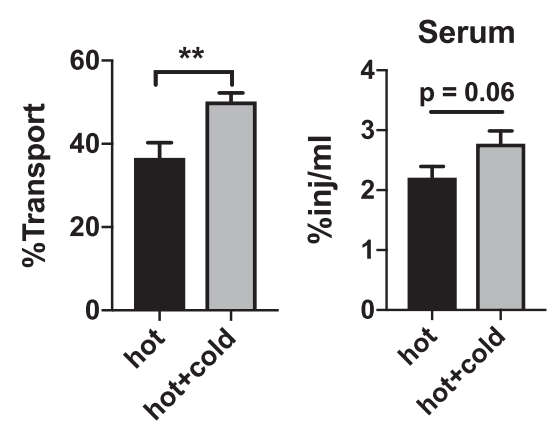

Fig. 7. Brain-to-blood efflux of C-Klep after its intracerebroventricular injection: (left-hand panel) percentage of the intracerebroventricularly injected material that was transported out of brain based on residual levels of radioactivity in brain; (right-hand panel) appearance of intracerebroventricular injection radioactivity in carotid artery serum. Both panels are consistent with increased efflux when C-Klep was coinjected with unlabeled Kleptose. $N=10$ /group.

An uptake of $0.06 \%$ of the administered dose could be sufficient for some effects in brain; for example, this is about four times more than the amount of morphine that accumulates in the brain and is in the range at which some regulatory proteins accumulate in the brain (Banks and Kastin, 1994).

These findings can be put in the context of the clinical experience with Niemann-Pick disease (Calias, 2017). A dose of $10 \mathrm{mg} / \mathrm{kg}$ given twice a week into the lateral ventricle of the brain produced some measures of CNS improvement, whereas peripheral infusions of $2.5 \mathrm{~g} / \mathrm{kg}$ given 2 to 3 times per week produced minor and transient improvements (Matsuo et al., 2013, 2014). The peripheral dose/intracerebroventricular dose ratio is thus 250:1. This is generally an adequate ratio for a substance that has CNS effects dependent on its ability to cross the BBB. It should be noted that this ratio is lower than predicted from the percentage of administered dose for the brain (the value here of $0.06 \%$ would predict that $1 / 0.0006=1666$ times the intracerebroventricular dose should be given) because not all of an intracerebroventricular dose is available to the brain. However, studies in rodents that produced robust CNS effects typically gave $4 \mathrm{~g} / \mathrm{kg}$ and did so early in the life of the animal when disease was perhaps not so established. Therefore, our studies suggest that possible reasons why peripheral dosing may be more effective in mice than in humans is that the peripheral dose given to humans is still about 2 to 3 times lower than needed, the brain-to-blood efflux system in humans may be more robust than in mice, or luminal surface sticking is higher in humans than rodents. These possibilities join the more traditional ones such as species differences in uptake, degradation, and brain distributions, and that therapy is begun later in the clinical course in humans than in laboratory animals.

Evidence shows that the BBB is altered in lysosomal storage diseases, including increased leakiness to circulating substances (Begley et al., 2008). It may be that in the disease condition CDs are able to take advantage of this disruption of the BBB, and can thus more easily access the CNS.

The uptake of C-Klep by all of the peripheral tissues examined exceeded that of the vascular marker, I-Alb. Also, all had measureable $K_{\mathrm{i}}$ values except for the heart. Given that the uptake by the heart greatly exceeded that of the vascular maker I-Alb, it is likely that the unidirectional efflux phase was too short to accurately measure. The percentage of the administered dose taken up per gram of tissue varied among the tissues from a low for liver of $0.187 \pm 0.045$ to a high for kidney of $5.90 \pm 1.57$. The extremes of these two tissues is consistent with excretion of cyclodextrins being entirely by kidney (Huang et al., 2016). Only kidney showed an effect of unlabeled Klep, which paradoxically increased kidney retention. This may reflect saturable reabsorption by the kidney, which would also explain the trend toward a decrease in serum as well. However, studies with fluorescein isothiocyanate-labeled cyclodextrins found no reabsorption in the proximal tubule (Huang et al., 2016).

In conclusion, we found that C-Klep can cross the BBB in small amounts and by a nonsaturable mechanism. However, uptake is characterized by very large variation among regions of the CNS and attenuated by a brain-to-blood efflux system. Furthermore, much of the apparent transport of C-Klep is actually material that is only loosely adhering to the luminal surface of the BBB. Thus, several factors determine the degree to which this cyclodextrin crosses the BBB. We conclude that the intravenous route is challenging, and to reach therapeutic levels in brain will likely require much higher peripheral doses (which could be limited by off-target toxicities) or a facilitating delivery system.

\section{Authorship Contributions}

Participated in research design: Banks, Engelke, Hansen, Bullock, Calias.

Conducted experiments: Hansen, Bullock.

Contributed new reagents or analytic tools: Engelke, Calias.

Performed data analysis: Banks, Hansen, Bullock.

Wrote or contributed to the writing of the manuscript: Banks, Engelke, Hansen, Bullock, Calias.

\section{References}

Banks WA, Fasold MB, and Kastin AJ (1997) Measurement of efflux rate from brain to blood, in Methods in Molecular Biology: Neuropeptides Protocols (Irvine GB and Williams CH eds) pp 353-360, Humana Press, Totowa, NJ.

Banks WA and Kastin AJ (1984) A brain-to-blood carrier-mediated transport system for small, N-tyrosinated peptides. Pharmacol Biochem Behav 21:943-946.

Banks WA and Kastin AJ (1985) Peptides and the blood-brain barrier: lipophilicity as a predictor of permeability. Brain Res Bull 15:287-292

Banks WA and Kastin AJ (1986) Modulation of the carrier-mediated transport of the Tyr-MIF-1 across the blood-brain barrier by essential amino acids. J Pharmacol Exp Ther 239:668-672.

Banks WA and Kastin AJ (1989) Quantifying carrier-mediated transport of peptides from the brain to the blood, in Methods in Enzymology, (Conn PM ed) vol. 168, pp 652-660, Academic Press, San Diego, CA.

Banks WA and Kastin AJ (1994) Opposite direction of transport across the bloodbrain barrier for Tyr-MIF-1 and MIF-1: comparison with morphine. Peptides 15: 23-29.

Banks WA, Kastin AJ, and Coy DH (1984) Evidence that $\left[{ }^{125} \mathrm{I}\right] \mathrm{N}-\mathrm{Tyr}$-delta sleepinducing peptide crosses the blood-brain barrier by a non-competitive mechanism Brain Res 301:201-207.

Banks WA, Kastin AJ, and Fasold MB (1988) Differential effect of aluminum on the blood-brain barrier transport of peptides, technetium and albumin. J Pharmacol Exp Ther 244:579-585.

Banks WA, Kastin AJ, Fischman AJ, Coy DH, and Strauss SL (1986) Carriermediated transport of enkephalins and N-Tyr-MIF-1 across blood-brain barrier. Am J Physiol 251:E477-E482.

Begley DJ, Pontikis CC, and Scarpa M (2008) Lysosomal storage diseases and the blood-brain barrier. Curr Pharm Des 14:1566-1580.

Blasberg RG, Fenstermacher JD, and Patlak CS (1983) Transport of $\alpha$-aminoisobutyric acid across brain capillary and cellular membranes. J Cereb Blood Flow Metab 3:8-32.

Calias P (2017) 2-Hydroxypropyl- $\beta$-cyclodextrins and the blood-brain barrier: considerations for Niemann-Pick disease type C1. Curr Pharm Des 23:6231-6238.

Conceicao J, Adeoye O, Cabral-Marques HM, and Lobo JMS (2018) Cyclodextrins as drug carriers in pharmaceutical technology: the state of the art. Curr Pharm Des 24:1405-1433.

Davidson CD, Ali NF, Micsenyi MC, Stephney G, Renault S, Dobrenis K, Ory DS, Vanier MT, and Walkley SU (2009) Chronic cyclodextrin treatment of murine Niemann-Pick C disease ameliorates neuronal cholesterol and glycosphingolipid storage and disease progression. PLoS One 4:e6951.

Davis ME and Brewster ME (2004) Cyclodextrin-based pharmaceutics: past, present and future. Nat Rev Drug Discov 3:1023-1035.

el Tayar N, Tsai RS, Testa B, Carrupt PA, and Leo A (1991) Partitioning of solutes in different solvent systems: the contribution of hydrogen-bonding capacity and polarity. J Pharm Sci 80:590-598. 
Frey WH II (2002) Bypassing the blood-brain barrier to deliver therapeutic agents to the brain and spinal cord. Drug Deliv Technol 2:46-49.

Gil ES, Wu L, Xu L, and Lowe TL (2012) $\beta$-Cyclodextrin-poly( $\beta$-amino ester) nanoparticles for sustained drug delivery across the blood-brain barrier. Biomacromolecules 13:3533-3541.

Huang J, Weinfurter S, Pinto PC, Pretze M, Kränzlin B, Pill J, Federica R, Perciaccante R, Ciana LD, Masereeuw R, et al. (2016) Fluorescently labeled cyclodextrin derivatives as exogenous markers for real-time transcutaneous measurement of renal function. Bioconjug Chem 27:2513-2526.

Kannan P, Schain M, Kretzschmar WW, Weidner L, Mitsios N, Gulyás B, Blom H, Gottesman MM, Innis RB, Hall MD, et al. (2017) An automated method measures variability in P-glycoprotein and ABCG2 densities across brain regions and brain matter. J Cereb Blood Flow Metab 37:2062-2075.

Kastin AJ, Fasold MB, and Zadina JE (2002) Endomorphins, Met-enkephalin, Tyr-MIF-1, and the P-glycoprotein efflux system. Drug Metab Dispos 30 $231-234$

Liu B, Turley SD, Burns DK, Miller AM, Repa JJ, and Dietschy JM (2009) Reversal of defective lysosomal transport in NPC disease ameliorates liver dysfunction and neurodegeneration in the npc1 $1^{-1-}$ mouse. Proc Natl Acad Sci USA 106: $2377-2382$.

Loftsson T and Brewster ME (2010) Pharmaceutical applications of cyclodextrins: basic science and product development. J Pharm Pharmacol 62: 1607-1621.

Maness LM, Banks WA, Podlisny MB, Selkoe DJ, and Kastin AJ (1994) Passage of human amyloid $\beta$-protein 1-40 across the murine blood-brain barrier. Life Sci $\mathbf{5 5}$ 1643-1650.

Maness LM, Kastin AJ, Banks WA, Banks MF, Podlisny MB, and Selkoe DJ (1993) Passage of human amyloid 1-40 across the murine blood-brain barrier (Abstract). Society for Neuroscience Abstracts 19:696.

Matsuo M, Shraishi K, Wada K, Ishitsuka Y, Doi H, Maeda M, Mizoguchi T, Eto J, Mochinaga S, Arima $\mathrm{H}$, et al (2014) Effects of intracerebroventricular administration of 2-hydroxypropyl- $\beta$-cyclodextrin in a patient with Niemann-Pick Type C disease. Mol Genet Metab Rep 1:391-400.
Matsuo M, Togawa M, Hirabaru K, Mochinaga S, Narita A, Adachi M, Egashira M, Irie T, and Ohno K (2013) Effects of cyclodextrin in two patients with NiemannPick Type C disease. Mol Genet Metab 108:76-81.

Monnaert V, Tilloy S, Bricout H, Fenart L, Cecchelli R, and Monflier E (2004) Behavior of $\alpha-, \beta$-, and $\gamma$-cyclodextrins and their derivatives on an in vitro model of blood-brain barrier. J Pharmacol Exp Ther 310:745-751.

Nonaka N, Farr SA, Kageyama H, Shioda S, and Banks WA (2008) Delivery of galanin-like peptide to the brain: targeting with intranasal delivery and cyclodextrins. J Pharmacol Exp Ther 325:513-519.

Nonaka N, Farr SA, Nakamachi T, Morley JE, Nakamura M, Shioda S, and Banks WA (2012) Intranasal administration of PACAP: uptake by brain and regional brain targeting with cyclodextrins. Peptides 36:168-175.

Patlak CS, Blasberg RG, and Fenstermacher JD (1983) Graphical evaluation of blood-to-brain transfer constants from multiple-time uptake data. $J$ Cereb Blood Flow Metab 3:1-7.

Pontikis CC, Davidson CD, Walkley SU, Platt FM, and Begley DJ (2013) Cyclodextrin alleviates neuronal storage of cholesterol in Niemann-Pick $\mathrm{C}$ disease without evidence of detectable blood-brain barrier permeability. J Inherit Metab Dis 36:491-498.

Soares AF, Carvalho RdeA, and Veiga F (2007) Oral administration of peptides and proteins: nanoparticles and cyclodextrins as biocompatible delivery systems. Nanomedicine (Lond) 2:183-202.

Triguero D, Buciak J, and Pardridge WM (1990) Capillary depletion method for quantification of blood-brain barrier transport of circulating peptides and plasma proteins. J Neurochem 54:1882-1888.

Varca GHC, Andréo-Filho N, Lopes PS, and Ferraz HG (2010) Cyclodextrins: an overview of the complexation of pharmaceutical proteins. Curr Protein Pept Sci 11: $255-263$.

Address correspondence to: W.A. Banks, University of Washington, VAPSHCS/GRECC S-182, Building 1, Room 810A, 1660 S. Columbian Way, Seattle, WA 98108. E-mail: wabanks1@uw.edu 